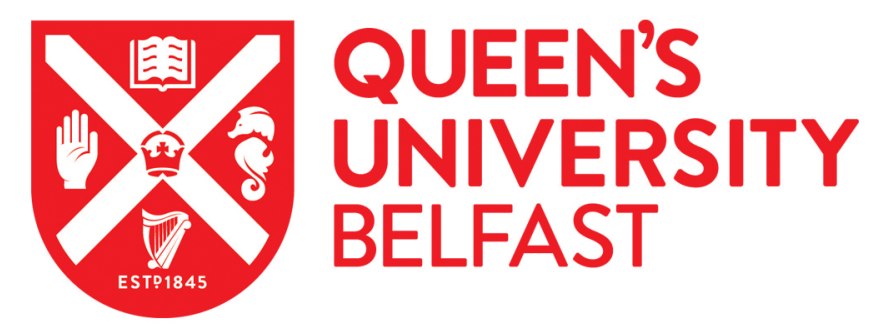

\title{
A Low Complexity Detector with MRC Diversity Reception for MCIK- OFDM
}

Chatziantoniou, E., Crawford, J., \& Ko, Y. (2016). A Low Complexity Detector with MRC Diversity Reception for MCIK-OFDM. In Proceedings of the IEEE 27th International Symposium on Personal, Indoor and Mobile Radio Communications Institute of Electrical and Electronics Engineers Inc..

https://doi.org/10.1109/PIMRC.2016.7794732

Published in:

Proceedings of the IEEE 27th International Symposium on Personal, Indoor and Mobile Radio Communications

Document Version:

Peer reviewed version

Queen's University Belfast - Research Portal:

Link to publication record in Queen's University Belfast Research Portal

Publisher rights

(c) 2016 IEEE.

This work is made available online in accordance with the publisher's policies. Please refer to any applicable terms of use of the publisher.

\section{General rights}

Copyright for the publications made accessible via the Queen's University Belfast Research Portal is retained by the author(s) and / or other copyright owners and it is a condition of accessing these publications that users recognise and abide by the legal requirements associated with these rights.

Take down policy

The Research Portal is Queen's institutional repository that provides access to Queen's research output. Every effort has been made to ensure that content in the Research Portal does not infringe any person's rights, or applicable UK laws. If you discover content in the Research Portal that you believe breaches copyright or violates any law, please contact openaccess@qub.ac.uk. 


\title{
A Low Complexity Detector with MRC Diversity Reception for MCIK-OFDM
}

\author{
Eleftherios Chatziantoniou, James Crawford, Youngwook Ko \\ Institute of Electronics, Communications and Information Technology \\ Queen's University of Belfast \\ NI Science Park, Belfast, BT3 9DT, United Kingdom \\ Email: \{1.chatziantoniou, jcrawford23,y.ko\}@qub.ac.uk
}

\begin{abstract}
Multi-carrier index keying (MCIK) is a recently developed transmission technique that exploits the sub-carrier indices as an additional degree of freedom for data transmission. This paper investigates the performance of a low complexity detection scheme with diversity reception for MCIK with orthogonal frequency division multiplexing (OFDM). For the performance evaluation, an exact and an approximate closed form expression for the pairwise error probability (PEP) of a greedy detector (GD) with maximal ratio combining (MRC) is derived. The presented results show that the performance of the GD is significantly improved when MRC diversity is employed. The proposed hybrid scheme is found to outperform maximum likelihood (ML) detection with a substantial reduction on the associated computational complexity.
\end{abstract}

Index Terms-Maximal ratio combining (MRC), multi-carrier index keying (MCIK), orthogonal frequency division multiplexing (OFDM).

\section{INTRODUCTION}

Multi-carrier transmission techniques have become the key technology for high data-rate, wideband wireless communications. Orthogonal frequency division multiplexing (OFDM) has been adopted by the majority of wireless communication standards such as IEEE 802.11, IEEE 802.16, and 3GPP's LTE-Advanced, due to its capability of combating intersymbol interference caused by frequency selective fading channels. A new variant of OFDM, known as multi-carrier index keying OFDM (MCIK-OFDM), has been recently proposed as a means of extending the conventional two dimensional $M$-ary signal constellations to a third dimension, which is the sub-carrier index [1], [2]. Similar to the spatial modulation (SM) concept [3], in every MCIK-OFDM transmission only a subset of sub-carriers is activated, according to the incoming data, to convey constellation symbols.

The use of the sub-carrier indices as an additional degree of freedom enables the transmission of extra information bits without any additional bandwidth and power requirements. As a result, MCIK-OFDM constitutes a promising modulation technique for providing high data-rate services especially for low-cost, energy constrained wireless systems such as deviceto-device (D2D) communications. MCIK-OFDM has attracted significant attention as it can provide a balanced trade-off between error performance and spectral efficiency [4]. To this end, different MCIK-OFDM transceiver architectures have been proposed and analyzed over different conditions includ- ing frequency selective fading, high mobility, and time varying channels [5].

Accurate detection of the sub-carrier state is a key aspect of MCIK-OFDM systems. Maximum-likelihood (ML) detection is considered as the optimal detection method and has been extensively used in MCIK-OFDM [6]. However, for the successful development of low-cost, energy efficient MCIK-OFDM D2D wireless systems, low complexity detection schemes are required. In this context, a low-complexity greedy detection method has been proposed in [7]. Unlike the ML detector, which performs an exhaustive search of all possible sub-carrier index combinations and their corresponding constellation symbols, the greedy detector (GD) first detects the active subcarriers, and then estimates their corresponding symbols.

The GD has been found to substantially reduce the computational complexity compared to ML detection. However, the use of the maximum received power as a test statistic to determine the active indices results to a diversity order, $d=1$. As a result, the GD fails to retain the same diversity order, i.e., $d=2$ as the ML detector, which in turn leads to inferior detection performance. In this work, we propose diversity reception as a means of improving the performance of the GD by increasing its diversity order. The key advantage of the proposed detection scheme is the performance improvement over conventional greedy detection without any additional computational cost. This approach results to a low complexity receiver architecture with comparable detection performance over ML, which is expected to facilitate the use of MCIKOFDM by low-cost wireless D2D systems. The performance analysis of the proposed scheme is based on a novel closedform expression for the average pairwise error probability (PEP) of greedy detection with maximal ratio combining (MRC). A closed-form expression that approximates the average PEP of greedy detection with MRC diversity reception (GD-MRC) is also derived. The approximate expression is found to provide acceptable accuracy levels with up to 3 $\mathrm{dB}$ difference. Furthermore, the symbol error rate (SER) performance of MCIK-OFDM with GD-MRC is investigated. Our simulation results show that for some cases MCIK-OFDM with GD-MRC can outperform conventional OFDM.

The rest of this paper is organized as follows. The fundamentals of MCIK-OFDM and the principle of the GD are described in Section II. Section III presents the concept of applying diversity reception in the GD. In Section III novel 
closed form expressions for the exact and approximate overall PEP for MCIK-OFDM with diversity reception are derived. The performance of the proposed scheme is analyzed in Section IV through analytical and simulation results. Finally, Section V summarizes and concludes the presented work.

\section{SySTEM MODEL}

\section{A. MCIK-OFDM}

Let us consider an MCIK-OFDM system with $N_{c}$ subcarriers that consists of $c$ clusters of $N$ sub-carriers, i.e, $N_{c}=c N$. In every transmission, only $K$ out of $N$ sub-carriers per cluster are randomly activated to deliver data symbols, while $N-K$ sub-carriers are zero padded. For each cluster, let $\mathbf{I}=\left\{i_{1}, \ldots, i_{K}\right\}$, where $i_{k} \in[1, \ldots, N]$ for $k=1, \ldots, K$ denote the indices of $K$ active sub-carriers. The corresponding block of symbols is denoted by $\mathbf{s}=\left[s_{(1)}, \ldots, s_{(K)}\right], s_{(k)} \in \mathcal{S}$. Using both $\mathbf{I}$ and $\mathbf{s}$, an MCIK-OFDM block is generated as $\mathbf{x}=[x(1), \ldots, x(N)]^{T}, x(\alpha) \in\{0, \mathcal{S}\}$ and $\alpha=1, \ldots, N$, where the indices of $N-K$ inactive sub-carriers are employed for additional data transmission.

For such an MCIK-OFDM, the total number of active subcarrier index combinations is $\left(\begin{array}{l}N \\ K\end{array}\right)$, but for simplicity and efficient mapping of the data bits, $2^{\left\lfloor\log _{2}\left(\begin{array}{l}N \\ K\end{array}\right)\right\rfloor}$ combinations are used, where $\left(\begin{array}{l}a \\ b\end{array}\right)$ and $\lfloor$.$\rfloor denote the binomial coefficient and the$ floor function, respectively. Therefore, in every MCIK-OFDM transmission $m_{1}=\left\lfloor\log _{2}\left(\begin{array}{l}N \\ K\end{array}\right)\right\rfloor$ bits are used to modulate the sub-carrier indices and $m_{2}=K \log _{2} M$ bits are transmitted via the corresponding $\mathrm{M}$-ary signal constellation.

Let the MCIK-OFDM block transmitted over a frequency selective Rayleigh fading. The channel fading coefficients can be defined as $\mathbf{H}=\operatorname{diag}(h(1), \ldots, h(N))$, where $h(\alpha)$ represents an independent and identically distributed (i.i.d.) Gaussian complex random variable with zero mean and unit variance, i.e., for $h(\alpha) \in \mathbf{I}, h(\alpha) \sim \mathcal{C N}(0,1)$, whereas for $h(\alpha) \notin \mathbf{I}, h(\alpha)=0$.

The input-output model of MCIK-OFDM is described as,

$$
\mathbf{y}=\mathbf{x H}+\mathbf{n},
$$

where $\mathbf{y}=[y(1), \ldots, y(N)], \mathbf{x}$ denotes an MCIK-OFDM signal block, and $\mathbf{n}=[n(1), \ldots, n(N)]$ is additive white Gaussian noise (AWGN), i.e., $n(\alpha) \sim \mathcal{C N}\left(0, N_{0}\right)$. The signal-to-noise ratio (SNR), is given by $E_{s} / N_{0}$, where $E_{s}$ denotes the power of each data symbol.

\section{B. Greedy Detector}

In MCIK-OFDM the receiver must follow a two-step detection process to first determine the indices of the active sub-carriers and subsequently detect their corresponding data symbols. In the first step, the GD measures the received power on each sub-carrier and estimates $K$ sub-carriers with the greatest received power as active. Hence,

$$
\hat{\alpha}=\underset{\alpha}{\arg \max }\left|y_{j}(\alpha)\right|^{2},
$$

where $y_{j}(\alpha)$ is the received signal from an MCIK-OFDM subcarrier with $\alpha=1, \ldots, N$, and $j=1, \ldots, K$ denotes an iteration count. This process is repeated with an increment of $j=j+1$ until $j=K$.

In the second step, ML is applied to the estimated active sub-carriers to detect the corresponding data symbols. Hence,

$$
\hat{s}(\hat{\alpha})=\underset{x(\hat{\alpha}) \in \mathcal{S}}{\arg \min }|y(\hat{\alpha})-h(\hat{\alpha}) x(\hat{\alpha})|^{2} .
$$

The performance of the GD is evaluated in terms of PEP, which expresses the probability of miss-detecting an inactive sub-carrier as active, i.e. $P(\alpha \rightarrow \tilde{\alpha})$. Given that GD uses the maximum received power as a test statistic, PEP is defined as the probability that the maximum noise power of a sub-carrier is greater than the power of an active one [7]. Formally,

$$
P(\alpha \rightarrow \tilde{\alpha})=P\left(\max \left\{\|n(\alpha)\|^{2}>\|h(\alpha) x(\alpha)+n(\alpha)\|^{2}\right) .\right.
$$

A closed-form expression for (4) has been derived in [7] as,

$$
\left.P(\alpha \rightarrow \tilde{\alpha})=1-\sum_{q=0}^{N-K}\left(\begin{array}{c}
N-K \\
q
\end{array}\right) \frac{-1^{q}}{q+1} e^{\gamma_{\alpha}\left(\frac{1}{q+1}-1\right.}\right) .
$$

where $\gamma_{\alpha}$ denotes the instantaneous SNR of an active subcarrier.

A much simpler expression that approximates (5) is derived in [8].

$$
P(\alpha \rightarrow \tilde{\alpha}) \simeq \frac{N-K}{2} e^{-\frac{\gamma_{\alpha}}{2}}
$$

For simplicity on the notations, the SNR of the active subcarrier, $\gamma_{\alpha}$ is denoted by $\gamma$ hereafter.

\section{Diversity Order}

For MCIK-OFDM with ML, the receiver makes a joint decision on the active sub-carriers and the constellation symbols. However, by considering only the active sub-carrier detection, the PEP of ML can be formulated as,

$$
\begin{aligned}
P(\alpha \rightarrow \tilde{\alpha}) & =P\left(\|h(\tilde{\alpha}) x+n\|^{2}>\|h(\alpha) x+n\|^{2}\right) \\
& =P(h(\tilde{\alpha}) x-h(\alpha) x>0) \\
& =P(x(h(\tilde{\alpha})-h(\alpha))>0)
\end{aligned}
$$

where $h_{\alpha}$ and $h_{\tilde{\alpha}}$ denote the channel amplitude of an active and an inactive sub-carrier, respectively.

Similarly, the PEP of GD is formulated as,

$$
\begin{aligned}
P(\alpha \rightarrow \tilde{\alpha}) & =P\left(\max \|n\|^{2}>\|h(\alpha) x+n\|^{2}\right) \\
& =P(\max \|n\|-\|h(\alpha) x+n\|>0)
\end{aligned}
$$

From (7) it can be seen that ML exploits the channel amplitude of both the active and inactive sub-carrier to determine the index of the active sub-carriers. As a result, the use of two sources of information results to a diversity order of 2 . On the other hand, with reference to (8), GD uses only the channel amplitude of the active sub-carrier. Hence, the use of one source of information from GD results to a diversity order of 1 . 


\section{GREedy Detector With Diversity ReCEPTION}

Diversity combining is a well known technique for mitigating the fading effects in wireless communication channels [10]. Based on the fact that independent signal paths have a low probability of experiencing deep fades simultaneously, receiver diversity combines the signal of multiple paths in a way that the fading of the resultant signal is reduced.

By employing diversity reception in the GD, the detection process can be described as,

$$
\hat{\alpha}=\underset{\alpha}{\arg \max }\left|z_{j}(\alpha)\right|^{2},
$$

where $z_{j}(\alpha)=x h_{\Sigma}+n$ with $h_{\Sigma}$ denoting the complex channel amplitude of the combined signal of $L$ individual signal paths.

The complex amplitude term, $h_{\Sigma}$, results in an SNR $\gamma_{\Sigma}$ at the combiner output, where its distribution depends on the number of diversity paths, the fading distribution on each path, and the combining technique. Following the same detection process as described in the previous subsection, the instantaneous PEP for GD with diversity reception is given by,

$$
P(\alpha \rightarrow \tilde{\alpha})=1-\sum_{q=0}^{N-K}\left(\begin{array}{c}
N-K \\
q
\end{array}\right) \frac{-1^{q}}{q+1} e^{\gamma_{\Sigma}\left(\frac{1}{q+1}-1\right)} .
$$

Similarly, using (6), the approximate instantaneous PEP for GD with diversity receptions is obtained by,

$$
P(\alpha \rightarrow \tilde{\alpha}) \simeq \frac{N-K}{2} e^{-\frac{\gamma_{\Sigma}}{2}} .
$$

\section{A. MRC Diversity Reception}

In MRC diversity combining $L$ individual signals from each diversity branch are first weighted by their signal voltage to noise power ratios and then summed coherently. Assuming perfect knowledge of the branch amplitudes and phases, MRC is considered as the optimal diversity scheme and therefore provides maximum performance improvement. For a Rayleigh distributed fading envelope the distribution of the SNR output at the combiner, $\gamma_{\Sigma}$, is chi-squared with $2 L$ degrees of freedom, $E\left[\gamma_{\Sigma}\right]=L \bar{\gamma}$, variance $2 L \bar{\gamma}$ and a probability density function (PDF) given as,

$$
f_{\gamma_{\Sigma}}(\gamma)=\frac{\gamma^{L-1} e^{-\frac{\gamma}{\gamma}}}{\bar{\gamma}^{L}(L-1) !} .
$$

where $\bar{\gamma}$ is the average SNR.

\section{B. Exact PEP}

The average PEP for GD-MRC over Rayleigh fading can be obtained by averaging (5) over (12). Formally,

$$
\begin{aligned}
P^{M R C}(\alpha \rightarrow \tilde{\alpha}) & =\int_{0}^{\infty}\left[1-\sum_{q=0}^{N-K}\left(\begin{array}{c}
N-K \\
q
\end{array}\right) \frac{-1^{q}}{q+1}\right. \\
& \left.\times e^{\gamma\left(\frac{1}{q+1}-1\right)}\right] \frac{\gamma^{L-1} e^{-\frac{\gamma}{\gamma}}}{\bar{\gamma}^{L}(L-1) !} d \gamma .
\end{aligned}
$$

The integral in (13) can be solved by using [9, eq. (3.3813)]. Hence,

$$
\begin{aligned}
P^{M R C}(\alpha \rightarrow \tilde{\alpha}) & =1-\sum_{q=0}^{N-K}\left(\begin{array}{c}
N-K \\
q
\end{array}\right) \frac{-1^{q}}{q+1} \\
& \times\left(\frac{q+1}{q(\bar{\gamma}+1)+1}\right)^{L} .
\end{aligned}
$$

A detailed description of the derivation is shown in the Appendix.

By using the law of total probability, the upper bound for the overall average PEP for MCIK-OFDM with GD-MRC over Rayleigh fading is given by,

$$
\begin{aligned}
P_{M R C} & \leq \frac{K}{N} \sum_{\alpha=1}^{N} P^{M R C}(\alpha \rightarrow \tilde{\alpha}) \\
& =K\left[1-\sum_{q=0}^{N-K}\left(\begin{array}{c}
N-K \\
q
\end{array}\right) \frac{-1^{q}}{q+1}\left(\frac{q+1}{q(\bar{\gamma}+1)+1}\right)^{L}\right] .
\end{aligned}
$$

\section{Approximate PEP}

Following the same approach as in the previous subsection, the approximate PEP for MCIK-OFDM with GD-MRC over Rayleigh fading is obtained by averaging (6) over (12) as,

$$
P^{M R C}(\alpha \rightarrow \tilde{\alpha}) \simeq \int_{0}^{\infty} \frac{N-K}{2} e^{-\frac{\gamma}{2}} \frac{\gamma^{L-1} e^{-\frac{\gamma}{\bar{\gamma}}}}{\bar{\gamma}^{L}(L-1) !} d \gamma .
$$

After solving the integral in (16) a closed-form expression for the approximate PEP for MCIK-OFDM with MRC diversity reception is given as,

$$
P^{M R C}(\alpha \rightarrow \tilde{\alpha}) \simeq \frac{N-K}{2 \bar{\gamma}^{L}}\left(\frac{\bar{\gamma}+2}{2 \bar{\gamma}}\right)^{L} .
$$

Hence, the upper bound for the overall approximate PEP for MCIK-OFDM with MRC diversity reception over Rayleigh fading is given by,

$$
P_{M R C} \leq K\left[\frac{N-K}{2 \bar{\gamma}^{L}}\left(\frac{\bar{\gamma}+2}{2 \bar{\gamma}}\right)^{L}\right] .
$$

\section{Numerical Results ANd Discussion}

We consider an MCIK-OFDM system with $N_{c}=128$ subcarriers grouped into clusters of size $N$ with a QPSK constellation $(M=4)$ unless otherwise stated. The performance of the GD is evaluated over Rayleigh fading channels in terms of average PEP and the effective SNR, i.e., $E_{s} / N_{0}$ on a cluster basis. Note that since PEP increases with the number of active sub-carrier index combinations it is maximized for $K=N / 2$ whereas it is minimized for $K=1$. Therefore, our analysis considers both the worst and best case scenarios for $N=8$, $K=4$ and $N=4$ and $K=1$, respectively.

Fig. 1 depicts the performance of three different detection schemes: 1) ML; 2) GD; 3) GD-MRC, for MCIK-OFDM with $N=4$ and $K=1$. It is clearly shown that ML outperforms conventional GD owing to the diversity order of 2, especially 
at high SNR regions. Indicatively, the maximum difference is observed for a PEP of $10^{-4}$, where the power gain of ML over the GD is $18 \mathrm{~dB}$. On the other hand, when MRC is applied it is observed that the SNR requirements of GD are significantly reduced by up to $50 \%$.

In terms of computational complexity, the number of metric combinations for GD scales to $K \times N \times M$ whereas for ML scales to $2\left[\log _{2}\left(\begin{array}{l}N \\ K\end{array}\right)\right\rfloor M^{K}$. Hence, for the considered scenario, i.e., $N=8$ and $K=4$ and $M=4$, ML requires 16384 metric combinations whereas GD requires 128 . Therefore, GD with diversity reception constitutes a promising solution for MCIKOFDM with large number of sub-carriers and higher order modulation schemes, where ML becomes impractical.

Fig. 2 shows the PEP performance of the GD-MRC with respect to the number of diversity branches, $L$, for an MCIKOFDM system with $N=4$ and $K=1$. It is shown that the detection performance is significantly improved as the number of diversity branches increases. For example, for a given PEP of $10^{-4}$ a power gain of up to $10 \mathrm{~dB}$ is observed between $L=2$ and $L=4$. Furthermore, the highest diversity gain of approximately $20 \mathrm{~dB}$ is observed from the no diversity case to the dual branch case.

Fig. 3 depicts the PEP performance of the GD-MRC combining and $N=8$ and $K=4$. It is shown that as the number of diversity branches increases, the difference between the required SNR for the best, i.e., $N=4 K=1$ and worst case, i.e., $N=8, K=4$ reduces. For example, for a PEP of $10^{-2}$ an $8 \mathrm{~dB}$ difference is observed between the best and worst case without diversity, which reduces to $2 \mathrm{~dB}$ when MRC with 4 branches is employed.

In Fig. 4 a comparison between the exact and approximate PEP expression for GD-MRC is depicted for MCIK-OFDM with $N=8$ and $K=4$ over Rayleigh fading. A maximum difference of $3 \mathrm{~dB}$ between the results of (15) and (18) is observed, which reveals the tightness of the approximation. Furthermore, it can be seen that as the number of diversity branches increases the approximation becomes tighter with less than $1 \mathrm{~dB}$ difference between the exact an approximate results.

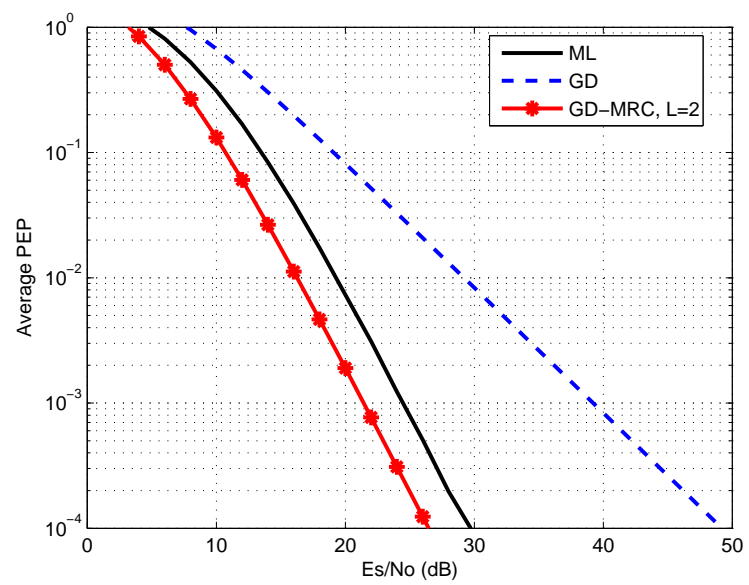

Fig. 1. PEP performance comparison between ML, GD and GD-MRC for MCIK-OFDM with $N=8$ and $K=4$.

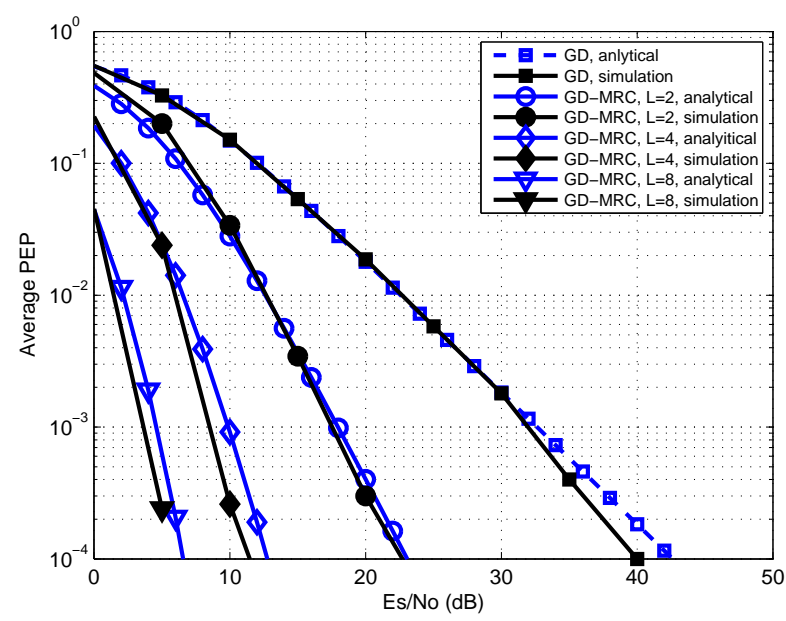

Fig. 2. PEP performance of MCIK-OFDM with MRC diversity reception for $N=4, K=1$ and varying $L$.

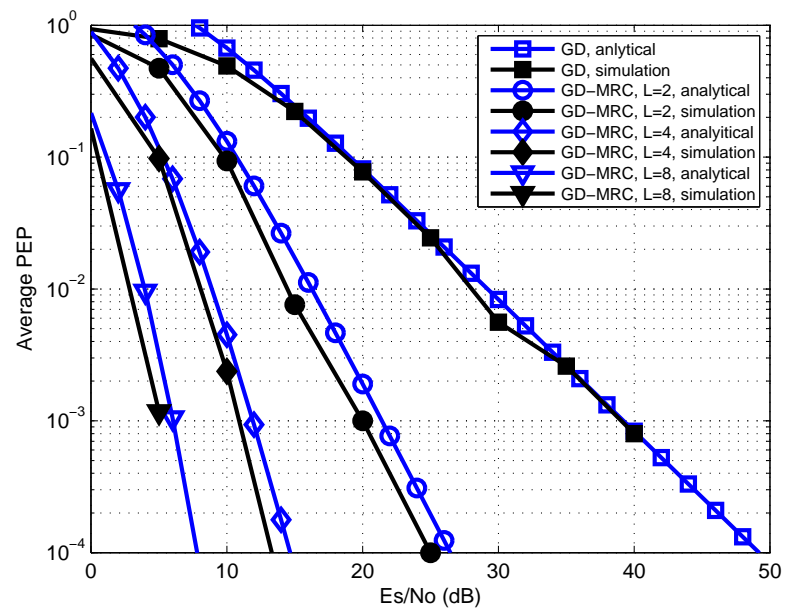

Fig. 3. PEP performance of MCIK-OFDM with MRC diversity reception for $N=8, K=4$ and varying $L$

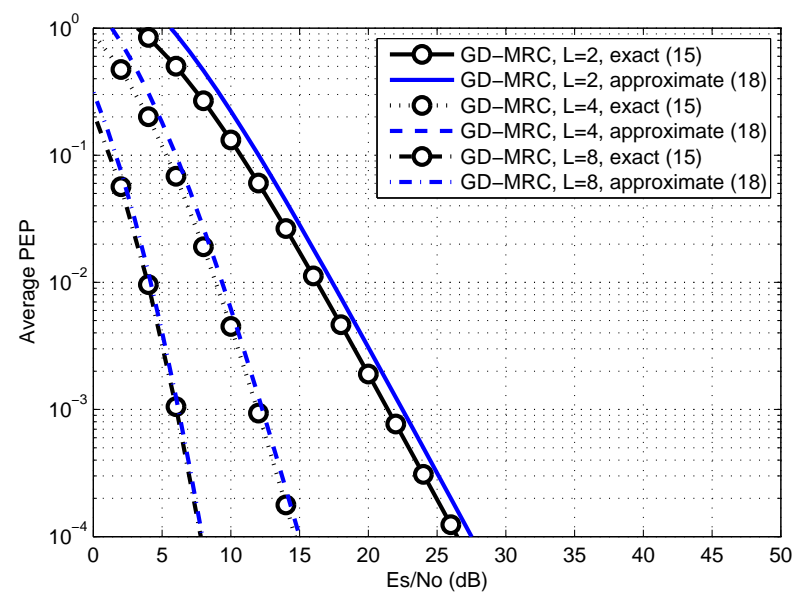

Fig. 4. Exact versus approximate PEP for MCIK-OFDM with MRC diversity reception for $N=8, K=4$ and varying $L$. 
Fig. 5 illustrates the SER performance of MCIK-OFDM with GD and GD-MRC over frequency selective Rayleigh fading. For a fair comparison the paramters of both systems are set to achieve the same spectral efficiency, i.e., 2 bits/Hz. The SER performance of conventional OFDM is compared with this of MCIK-OFDM for two different power allocation strategies: 1) constant power allocation (CPA) 2) uniform power allocation (UPA). In MCIK-OFDM with CPA the total transmit power is set as $K E_{s}$, whereas in UPA strategy the total transmit power is adjusted based on the ratio of the total to active sub-carrier, i.e., $(N / K) E_{s}$. It is shown that the error performance of MCIK-OFDM is improved with UPA over CPA with a power gain of approximately $1 \mathrm{~dB}$. It is also shown that MCIK-OFDM with MRC significantly improves the error performance by up to $4 \mathrm{~dB}$. More importantly, it is shown that MCIK-OFDM with a dual branch MRC can outperform classical OFDM by up to $0.5 \mathrm{~dB}$.

\section{CONCLUSiON}

In this paper diversity reception has been proposed as a means of improving the performance of a low complexity detection scheme for MCIK-OFDM. A novel closed-form expression for the average PEP of greedy detection with MRC diversity reception is derived. This expression is used to analyze the performance of MCIK-OFDM with diversity reception over Rayleigh fading channels. Furthermore, a closedform expression based on the approximate PEP has been derived. The obtained expression reduces the computational complexity by achieving accuracy levels of less than $1 \mathrm{~dB}$. Our results indicate that the greedy detection scheme can achieve similar performance to the ML detector with no additional computational complexity. Our future work will focus on applying different diversity combining techniques to the GD, and extending the analysis to account for SER.

\section{APPENDIX A}

\section{DERIVATION OF (14)}

Expression (13) can be rewritten as,

$$
\begin{gathered}
P^{M R C}(\alpha \rightarrow \tilde{\alpha})=\frac{1}{\bar{\gamma}^{L}(L-1) !}[\underbrace{\int_{0}^{\infty} \gamma^{L-1} e^{-\frac{\gamma}{\gamma}} d \gamma}_{I_{1}} \\
-\sum_{q=0}^{N-K}\left(\begin{array}{c}
N-K \\
q
\end{array}\right) \frac{-1^{q}}{q+1} \underbrace{\int_{0}^{\infty} \gamma^{L-1} e^{-\gamma\left(1-\frac{1}{q+1}+\frac{1}{\gamma}\right)} d \gamma}_{I_{2}}] .
\end{gathered}
$$

It can be seen that (13) includes 2 integrals of the same form, i.e., $\int_{0}^{\infty} x^{\nu-1} e^{-\mu x} d x$, which can be solved using [9, eq. (3.381-3)]. Hence,

$$
\begin{gathered}
I_{1}=\bar{\gamma}^{L} \Gamma(L) \\
I_{2}=\left(\frac{\bar{\gamma}(q+1)}{q(\bar{\gamma}+1)+1}\right)^{L} \Gamma(L)
\end{gathered}
$$

By substituting (A.2) and (A.3) into (A.1) and taking into account that $\Gamma(L)=(L-1)$ !, (A.1) can be simplified to (14).

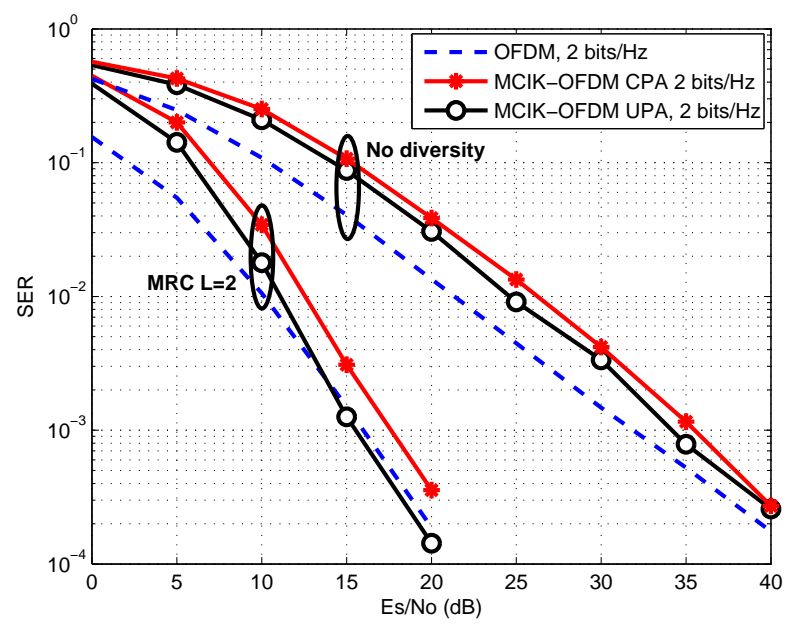

Fig. 5. SER performance of MCIK-OFDM with $N=8, K=6, M=4$ versus OFDM with $N=8, M=4$ with two branch MRC diversity reception.

\section{ACKNOWLEDGMENT}

This work has been supported by the Engineering and Physical Sciences Research Council (EPSRC) grant with reference number EP-M015521-1.

\section{REFERENCES}

[1] Abu-alhiga, R. and Haas, H., "Subcarrier-index modulation OFDM," in Proc. IEEE Pers., Indoor, Mobile Radio Commun., pp.177-171 Sep. 2009.

[2] Tsonev, D. and Sinanovic, S. and Haas, H., "Enhanced subcarrier index modulation (SIM) OFDM,"in Proc. IEEE GLOBECOM Workshops, pp.728-732, Dec. 2011.

[3] R. Mesleh, H. Haas, S. Sinanovic, C. W. Ahn, and S. Yun, "Spatial modulation," IEEE Trans. Veh. Technol., vol. 57, pp. 2228-2241, July 2008.

[4] E. Basar, U. Aygolu, E. Panayirci, and H. Poor, "Orthogonal frequency division multiplexing with index modulation," IEEE Trans. Signal Process., vol. 61, pp. 5536-5549, Nov. 2013.

[5] Basar, E. and Aygolu, U. and Panaylrcl, E, "Orthogonal frequency division multiplexing with index modulation in the presence of high mobility,"in Proc. Commun. and Netw., pp.147-151, Jul. 2013.

[6] Y. Ko, "A tight upper bound on bit error rate of joint OFDM and multicarrier index keying,'IEEE Commun. Lett. vol. 18, pp. 1763-1766, Oct. 2014.

[7] J. Crawford and Y. Ko, "Low complexity greedy detection method with generalized multicarrier index keying OFDM," in Proc. IEEE Pers., Indoor, Mobile Radio Commun., pp.688-693, Aug. 2015.

[8] E. Chatziantoniou, J. Crawford and Y. Ko "Performance analysis of a low complexity detector for MCIK-OFDM over TWDP fading," IEEE Commun. Lett, submitted Jan. 2016.

[9] I. Grandshteyn and I. Ryzhik, Table of Integrals, Series, and Products, 7th ed. Academic Press, 2007.

[10] A. Goldsmith, Wireless Communications. New York, NY, USA: Cambridge University Press, 2005. 